\title{
ARTICLE \\ Microfluidic actuators based on temperature-responsive hydrogels
}

\author{
Loïc D'Eramo ${ }^{1}$, Benjamin Chollet ${ }^{2}$, Marie Leman ${ }^{3}$, Ekkachai Martwong ${ }^{2}$, Mengxing Li $^{2}$, Hubert Geisler ${ }^{1}$, Jules Dupire ${ }^{3}$, \\ Margaux Kerdraon ${ }^{1}$, Clémence Vergne ${ }^{1}$, Fabrice Monti ${ }^{1}$, Yvette Tran $^{2}$ and Patrick Tabeling ${ }^{1}$
}

The concept of using stimuli-responsive hydrogels to actuate fluids in microfluidic devices is particularly attractive, but limitations, in terms of spatial resolution, speed, reliability and integration, have hindered its development during the past two decades. By patterning and grafting poly( $N$-isopropylacrylamide) PNIPAM hydrogel films on plane substrates with a $2 \mu \mathrm{m}$ horizontal resolution and closing the system afterward, we have succeeded in unblocking bottlenecks that thermo-sensitive hydrogel technology has been challenged with until now. In this paper, we demonstrate, for the first time with this technology, devices with up to 7800 actuated micro-cages that sequester and release solutes, along with valves actuated individually with closing and opening switching times of $0.6 \pm 0.1$ and $0.25 \pm 0.15 \mathrm{~s}$, respectively. Two applications of this technology are illustrated in the domain of single cell handling and the nuclear acid amplification test (NAAT) for the Human Synaptojanin 1 gene, which is suspected to be involved in several neurodegenerative diseases such as Parkinson's disease. The performance of the temperature-responsive hydrogels we demonstrate here suggests that in association with their moderate costs, hydrogels may represent an alternative to the actuation or handling techniques currently used in microfluidics, that are, pressure actuated polydimethylsiloxane (PDMS) valves and droplets.

Keywords: actuator; high throughput; hydrogel; microfluidics; single cell; valve

Microsystems \& Nanoengineering (2018) 4, 17069; doi:10.1038/micronano.2017.69; Published online: 1 January 2018

\section{INTRODUCTION}

Over the past 20 years, much progress has been made in the handling of liquids at the microscale. Since Terry's valve (1979) ${ }^{1}$, tens of micro-actuators of different types have been invented ${ }^{2}$. Pressure actuated polydimethylsiloxane (PDMS) microvalves ${ }^{3}$ deserve particular attention because, owing to their simplicity, thousands of units can be integrated on a single device, linking microfluidics and microelectronics ${ }^{4}$. PDMS valves currently represent an important tool for biology research ${ }^{5-8}$. In addition, dropletbased technology offers new methods of fluid manipulation, which are particularly powerful when high throughput is required $^{9-11}$. These two technologies complement each other: one is focused on an exquisite manipulation of fluids; the other is focused on high throughput performance. Nevertheless, limitations exist in terms of cost, material compatibility, functionality and automation ${ }^{12}$ such that new ideas and concepts are required in this area. The list of microfluidic applications has yet to be established with the existing actuators, and there is a need to develop complementary or alternative technologies that can contribute to fulfilling this list.

In this context, responsive hydrogels represent an interesting technological pathway. Their physical principle relies on their ability to undergo large volume changes when a stimulus (for example, thermal, photonic, chemical or electrical) is applied. The potential of these hydrogels is important because hydrogels possess properties ${ }^{13-16}$ (for example, adhesion, permeability, filtering, and chromatography) that coupled to their capacity of being actuated, enrich the number of functionalities that can be developed by each actuator, which in turn may considerably facilitate the design of microfluidic devices along with reducing their cost. However, hydrogel-based actuation technology is still at the early stage of development. The first microfluidic actuators realized with these materials were reported at the turn of the century ${ }^{17}$; since then, numerous devices have been fabricated: pumps (coupled to membranes) ${ }^{18-23}$, reconfigurable systems ${ }^{24}$, drug delivery devices ${ }^{25}$, self-regulating systems ${ }^{26}$, gel-based electroactive ${ }^{27}$ or photoactive valves ${ }^{28}$, and MEMS-coupled active mixers ${ }^{29}$. Despite multiple laboratory projects and twenty years of research, several bottlenecks restrict the technology from developing: mediocre spatial resolution, limited miniaturization capabilities, long time responses (usually more than $10 \mathrm{~s}$ ), complexity in the fabrication process and poor control of the physicochemistry $^{30,31}$. The last point is crucial because it raises concerns about the reliability of the technology ${ }^{32,33}$. In this article, we unblock these bottlenecks by adopting a strategy that is different from those of previous investigators. Our strategy is based on ex situ photopatterning with simultaneous cross-linking, grafting and patterning of the hydrogel prior to closing the device. PNIPAM photopatterning is not new ${ }^{34}$, but it has never been used to make microfluidic devices. With this approach, we can raise the microfabrication technique to a level where the fabrication of a variety of devices becomes feasible under excellent resolution and control conditions. These features are demonstrated with the realization of micrometric valves and cages enclosed by actuated

\footnotetext{
${ }^{1}$ MMN, IPGG, Microfluidique, MEMS, Nanostructures (MMN), Institut Pierre-Gilles de Gennes (IPGG), Gulliver, Ecole Supérieure de Physique et Chimie Industrielles (ESPCI Paris), PSL Research University, 10 rue Vauquelin, Paris 75005, France; ${ }^{2}$ SIMM, ESPCI Paris, Sciences et Ingénierie de la Matière Molle (SIMM), Ecole Supérieure de Physique et Chimie Industrielles (ESPCI Paris), PSL Research University, 10 rue Vauquelin, Paris 75005, France and ${ }^{3}$ Microfactory, 8 rue Jean Calvin, Paris 75005 , France

Correspondence: Yvette Tran or Patrick Tabeling (yvette.tran@espci.fr or patrick.tabeling@espci.fr)

Received: 1 February 2017; revised: 25 July 2017; accepted: 29 July 2017
} 
walls, in small or large quantities, the latter (caging/release) representing a new functionality for microfluidic technology. Biological applications in the domain of single cell manipulation and NAAT (Nuclear Acid Amplification Test) are illustrated.

\section{MATERIALS AND METHODS \\ Photo-patterning of hydrogel films}

A temperature-responsive hydrogel (poly( $N$-isopropylacrylamide), PNIPAM) is covalently grafted onto a flat substrate that forms the bottom wall of the microfluidic device. This process is an ex situ synthesis. Specifically, the hydrogel 'grafting onto' synthesis consists of simultaneous surface-grafting and cross-linking functionalized polymers through thiol-ene click chemistry. The surface-attached hydrogel films are obtained by coating the enefunctionalized polymers in the presence of dithiol molecules as cross-linkers on thiol-modified substrates. One advantage of this process is that it has been shown to allow both chemical crosslinking of the polymer chains and their covalent attachment to the surface with accurate and systematic control ${ }^{35}$. Ene-modified PNIPAM is dissolved in a 1:1 mixture of methanol and butanol at $10 \mathrm{wt} \%$. The cross-linker, dithioerythritol, is later added to this solution at $6 \mathrm{wt} \%$.

Patterning of the hydrogel is accomplished by exposure to deep UVs $(\sim 250 \mathrm{~nm})$. This can be achieved either by using standard photolithographic techniques, that is, with masks and UV lamps (8 Watt, $254 \mathrm{~nm}$ wavelength), or with a direct laser writing equipment (Dilase650 Kloe, Montpellier, France) using a $266 \mathrm{~nm}$ wavelength and $50 \mathrm{~mW}$ output-power source. After exposure, the non-attached polymer chains are removed by washing (with ultrapure water). The lateral resolution is $5 \mu \mathrm{m}$ with the standard lithographic technique, whereas it is $2 \mu \mathrm{m}$ with the direct laser writing. These scales control the minimum size of the structures we produce. In all cases, the height of the patterned structures can be selected to be between $100 \mathrm{~nm}$ and $7 \mu \mathrm{m}$.

\section{Preparation of thiol-modified substrates and integration of microheaters}

In the first version, called Method 1, a thiol-modified glass substrate was used. Specifically, the glass slides are cleaned with a Piranha solution (3:1 mixture of sulfuric acid and hydrogen peroxide heated at $200{ }^{\circ} \mathrm{C}$ for $20 \mathrm{~min}$ ). Silanization with 3mercaptopropyltrimethoxysilane is carried out on the freshly cleaned substrates to perform the thiol-modification of the surface. A solution of anhydrous toluene with a 3 vol\% of mercaptopropyltrimethoxysilane (ABCR Gelest) is put in contact with the substrates for $3 \mathrm{~h}$ inside a reactor under nitrogen. The solid substrates are rinsed and sonicated in toluene and finally dried with a nitrogen flow. In this version, the hydrogel is heated by placing the entire device on a PTC-200 Peltier thermocycler (MU Research) and applying a thermal paste (Radiospares) between the support and the glass slide of the microfluidic device to improve thermal contact.

In the second version, called Method 2, the hydrogels are locally heated by depositing a patterned chromium/gold bilayer onto the flat substrate. Glass wafers $\left(2^{\prime \prime}\right)$ are coated with a $100 \mathrm{~nm}$ layer of gold above a few nanometer-thick chromium adhesion layer. A layer of S1813 G2 (Shipley) is spin-coated and processed with a standard photolithography protocol to pattern the micro-heater structure. To ensure adhesion of the gel to the gold, a thiol modification of the surfaces is performed by immersing the gold substrates in a $1 \mathrm{~mm}$ solution of dithioerythritol (Sigma-Aldrich, St Louis, MO, USA) in chloroform for $2 \mathrm{~h}$ followed by rinsing in clear chloroform and sonicating for one minute. COMSOL numerical studies show that the temperature field extends around the heater for $\sim 50 \mu \mathrm{m}$, independently of the temperature.

\section{Closure of the device}

Two materials are used for closing the systems and producing the microfluidic devices. The first one is PDMS. In this case, the chips are fabricated following the standard soft lithography technique ${ }^{36}$. Bonding between the flat substrate and the PDMS chip is achieved by using an $\mathrm{O}_{2}$ plasma treatment for $45 \mathrm{~s}$. The second material used is the Norland Optical Adhesive (NOA 81 (ThorLabs)). In the case of NOA, the microfluidic stickers procedure is used ${ }^{37}$. NOA chips are obtained by stamping uncured NOA with a PDMS stamp and cured with UV exposure (LC8, Hamamatsu). Bonding between the substrate and the NOA chip is achieved with the presence of an uncured residual film after the first UV exposure. Sealing is then strengthened by a second UV exposure followed by a 30 -minute curing at $90^{\circ} \mathrm{C}$.

\section{Control of the hydrogel film along with its functionality}

The functionality of the hydrogel is evaluated by ellipsometric measurements of the swelling ratio (ratio between the hydrogel thickness in water and the dry thickness in air) performed at the polymer synthesis stage. The thickness of the hydrogel films is measured with a spectroscopic ellipsometer Nanofilm EP3 (Accurion $\mathrm{GmbH}$, Goettingen, Germany). Thicknesses range between $100 \mathrm{~nm}$ and $7 \mu \mathrm{m}$. Following dry thickness measurements, measurements in water are performed using a temperature-controllable liquid cell with thin glass walls fixed perpendicularly to the light path (the angle of incidence is fixed at $60^{\circ}$ ). The swelling ratio is the same (value of 1.5) for all PNIPAM films above the Lower Critical Solution Temperature LCST. Below the LCST, the value of 4 has been obtained with $2 \%$ ene-reactive polymers $^{38-40}$. After closure of the microfluidic devices, the in-chip functionality of the hydrogel valves is confirmed by hydrodynamic measurements using a MFCS Fluigent system equipped with a flow-rate device and determining the hydrodynamic resistance of the device at various temperatures.

\section{DNA amplification}

For amplification experiments, we use RCA amplification of a target Human Synaptonjanin 1 gene, which is considered to be potentially involved in Parkinson's disease. The amplification is performed with the Illustra Genomiphi V2 kit (GE Healthcare, Little Chalfont, UK). The amplification mixture is supplemented with $1 \mu \mathrm{l}$ of a qPCR gene expression primer/probe set (ThermoScientific, SYNJ1 FAM-MGB, 20×). Here the fluorescent probe acts as a hydration probe rather than a Taqman probe. The DNA concentration is $84 \mathrm{pg} \mathrm{\mu l}^{-1}$, corresponding statistically to 7 DNA molecules per $8 \mathrm{pl}$ hydrogel cage. The microchip is first filled with a solution of Pluronic F127 (2\%, Sigma Aldrich) and cycled between $20^{\circ} \mathrm{C}$ and $50^{\circ} \mathrm{C}$ on a PTC-200 Peltier device for $1 \mathrm{~h}$. The amplification mixture is then injected with an MFCS pressure controller (Fluigent) at $40^{\circ} \mathrm{C}$ and the temperature was switched to $28^{\circ} \mathrm{C}$ to begin the $2 \mathrm{~h}$ long incubation. The time between the end of the sample preparation and the beginning of the incubation is less than $5 \mathrm{~min}$. Control experiments are performed in parallel. Hydrogel cage fluorescence is monitored using a shuttered UV lamp and a camera as described in the following section.

\section{Optical observation and in situ determination of the hydrogel landscape}

The optical observations are performed with various techniques: interferometric microscopy, bright field microscopy or epifluorescence microscopy, using inverted microscopes (usually with $2.5 \times$ or $5 \times$ objectives) or a $0.8-9.2 \times$ binocular microscope. For fluorescence studies, the dyes are $0.8 \mathrm{~mm}$ solution of fluorescein in Milli-Q water and $0.1 \mathrm{~mm}$ solution of rhodamine $B$ and we used two-color microscopy. Images are taken using sensitive cameras (Hamamatsu ImageEM and OrcaER) and processed using Matlab software. 
To measure the hydrogel topography in situ, we use a fluorescence-based technique that consists of filling the device with a dilute fluorescein solution and measuring the intensity field. Supplementary Information 2 shows that in the range of the concentration used, this intensity is proportional to the difference between the hydrogel and the channel heights. By converting intensities into heights, we determine the topography of the hydrogel structure in situ in real time with an accuracy estimated to be in the order of 1-2 microns (for experiments lasting several seconds). This technique allows direct visualization of the dynamics of the hydrogel actuators.

\section{RESULTS}

\section{Hydrogel-based valving}

Micro-valving is a fundamentally important microfluidic functionality that is well achieved with our hydrogel technology. The fabrication process of a microfluidic valve is shown in Figure 1 a. We integrate a rectangular patch of gel, similar to Figure $1 \mathrm{~b}$ (upper figure), in a micro-channel normal to its length using the NOA closure technique explained previously. To seal the valve, the hydrogel patch length slightly exceeds the channel width. Closing is achieved by applying a force of a few newtons on the material that is pressed onto the bottom plate to close the channel so that the excess hydrogel is squeezed between this material and the bottom plate. This construction prevents the presence of dead volumes and the advent of leaks. Figure 1c shows the fluorescence microscopy images and the corresponding topographies of a valve in the open and closed positions (see Materials and Methods, and Supplementary Information 2). At $40^{\circ} \mathrm{C}$, that is, above the LCST, the valve is open. The gel occupies a fraction of the microchannel height, or $40 \%$ in this particular case. As the temperature is lowered to $25^{\circ} \mathrm{C}$, the hydrogel film swells and the valve closes. Supplementary Information 3 shows the movie corresponding to Figure $1 \mathrm{c}$.

Physically, the closure of the valve is a result of the considerable increase of the local hydrodynamic resistance caused by the obstruction of the channel with the hydrogel. The obstruction is due to the design (the channel or chamber height is $80-90 \%$ of the height of the swollen gel in free conditions). Diffusion measurements are presented later in the paper. In such conditions, calculations based on hydrodynamics show the increase of resistance between the open and the closed states on the order of $(\mathrm{h} / \mathrm{r})^{4}$, where $h$ is the channel height and $r$ is the pore size. With $h \sim 10 \mu \mathrm{m}$ and $r \sim 1 \mathrm{~nm}$, this ratio is on the order of $10^{16}$. In such conditions, without diffusion, it would take years for a water molecule to cross the gel barrier under the action of a pressure gradient. For solutes of comparable size or larger than the gel pore, this time is much longer. In all cases, the increase of resistance is so large that the circuit can be considered hydrodynamically closed. This does not preclude diffusive exchanges across the gel barrier, which will be presented in the following section.

Figure 2a presents the temporal evolution of the flow-rate, for an isolated valve subjected to a constant pressure of $500 \mathrm{mbar}$ and actuated periodically in time, with a period of $5 \mathrm{~min}$; the experiments lasted for $10 \mathrm{~h}$. Beyond demonstrating that the actuation is reproducible, the graphs show that leaks are so small that they are not measurable. Further studies show that in this system, the burst pressure (that is, the pressure above which closed valves are forced to open), is above three bars, a figure well compatible with the vast majority of microfluidic applications. Added to these characteristics, one must recall the absence, by construction, of dead volumes. The micro-valves we report here therefore possess excellent performance.

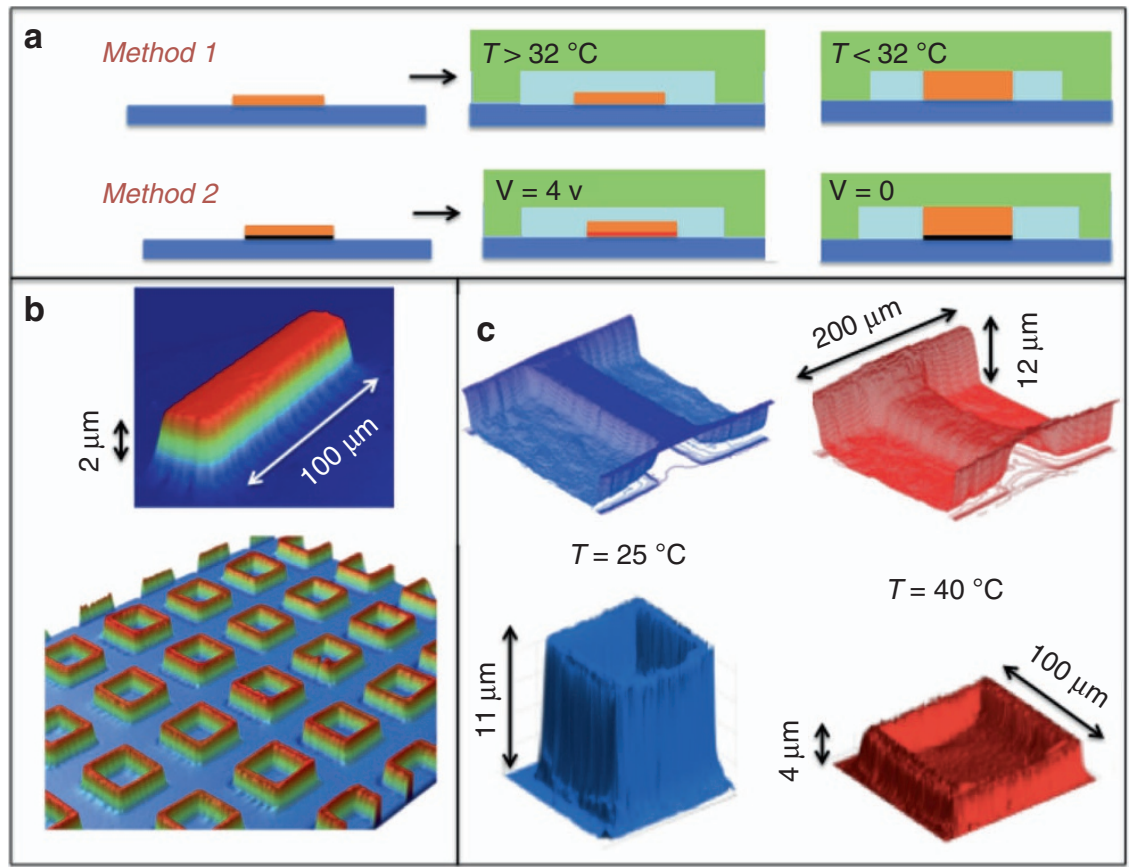

Figure 1 (a) Microfabrication technique: hydrogel structures are deposited and grafted/patterned on the substrate. With Method 1, the temperature changes are global, whereas with Method 2, hydrogel structures are actuated individually as a result of the deposition of the patterned Chromium/Gold layers. Then, the system is closed with a NOA or PDMS structure. At low temperatures (T < LCST), the hydrogel is swollen. By raising the temperature above the LCST or by heating up the metal layer, the hydrogel collapses. (b) Various surface-attached hydrogel patterns visualized with interferometric microscopy: Individual patch and array of square microcages. The widths of the structures (patch and microcage walls) are $10 \mu \mathrm{m}$, the cage (horizontal) size is $200 \mu \mathrm{m} \times 200 \mu \mathrm{m}$, the wall height is $2 \mu \mathrm{m}$. (c) In situ topographic images of valves and cages (Supplementary Information 2) using false colors to illustrate the thermal conditions. At $25^{\circ} \mathrm{C}$, the hydrogel is swollen, the valve is closed and the cage walls are raised. At $40^{\circ} \mathrm{C}$, the hydrogel is collapsed, the valve is open and chamber walls are in a low position. 


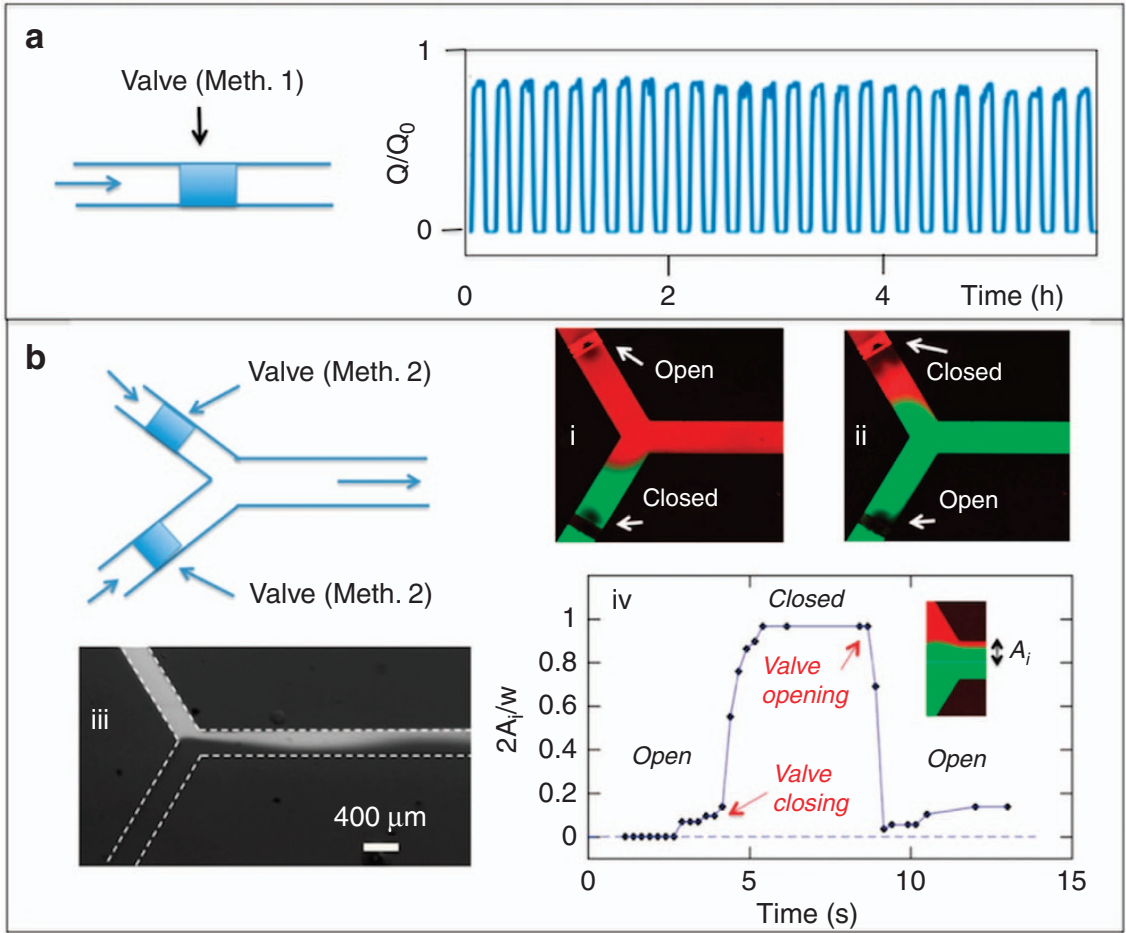

Figure 2 (a) Temporal evolution of the flow-rate $Q$ in a $9.4 \mu \mathrm{m} \times 400 \mu \mathrm{m} \times 1.2 \mathrm{~cm}$ channel integrating a hydrogel valve, using Method 1 , and subjected to a temperature field switching between 25 and $50^{\circ} \mathrm{C}$, at a frequency of $1.4 \mathrm{mHz}$. $Q_{0}$ is $7 \mu \mathrm{l} / \mathrm{mn}$. (b) Device with two locally actuated hydrogel valves, elaborated according to Method 2. The channel widths are $400 \mu \mathrm{m}$ (i): with the upper valve open and the lower valve closed, the red dye flows downstream. (ii) With the upper valve closed and the lower valve open, the green dye passes. (iii) Typical fluorescence image for two valves alternatively open and close at a $2 \mathrm{~Hz}$ frequency (Method 2). (iv) Evolution of the interface position Ai, that is, its distance from the symmetry axis of the collecting channel (see $\mathrm{d}$, insert), and divided by half the channel width $w \approx 400 \mu \mathrm{m}$. Ai is measured as a function of time, with the lower (green) valve maintained open, and the upper (red) valve, subjected to a sequence of heating events. The (red) valve is initially heated above $32{ }^{\circ} \mathrm{C}$. Then at $t=4.2 \pm 0.1 \mathrm{~s}$, heating is turned off; finally, at $t=8.9 \pm 0.1 \mathrm{~s}$, heating is turned on again. From d, one estimates that the closing and opening times of the valve are, respectively, $0.6 \pm 0.1 \mathrm{~s}$ and $0.25 \pm 0.15 \mathrm{~s}$.

Figure $2 \mathrm{~b}$ represents devices with two valves actuated independently (see Supplementary Information 4 for detail). They are designed to measure the switching time and demonstrating that fluids can be distributed in a microfluidic system with a local control. In the resting state when no local heating is applied (room temperature $21^{\circ} \mathrm{C}$ ), the valves are closed, while they open independently when local heating is applied. For the sake of visualization, the two aqueous solutions driven in the system are labeled, respectively, by fluorescein and rhodamine, and observed with a two-color microscope. Figure $2 \mathrm{~b}$ shows that, as expected, two different fluids can be transported sequentially in the same collecting channel, by actuating two valves. In Figure $2 \mathrm{~b}$ (iii), two aqueous solutions are injected, one is a $0.8 \mathrm{mmol} \mathrm{I}^{-1}$ fluorescein solution and the other is pure water. The two valves are actuated with a $180^{\circ}$ phase shift, at a frequency of $2 \mathrm{~Hz}$. In such circumstances, fluorescein waves develop. They are advected by the main stream and propagate downstream (see Supplementary Information 4). Measurements of the amplitude of these waves, plotted as a function of the frequency, exhibit a low pass-band behavior, with a cutoff frequency of $5 \mathrm{~Hz}$. The switching time of the valves can in fact be inferred from Figure $2 \mathrm{~b}$ (iv). This figure, extracted from the video corresponding to Figure $2 \mathrm{~b}$ (i), (ii), shows the temporal evolution of the green/red interface position $A i$, divided by half the channel width $W$, as the upper (red) valve is subjected to sudden heating of the micro-heater, or to sudden turn off of the heating. In all these experiments, the lower (green) valve is maintained open. Initially, the (red) valve is heated above $32{ }^{\circ} \mathrm{C}$, the two valves are thus open and consequently the red/green interface lies at the center of the channel, for which $A i=0$. At $t=4.2 \pm 0.1 \mathrm{~s}$, the heating of the red valve is turned off, the gel swells, the (red) valve closes, and the interface displaces rapidly towards the channel wall, for which $\frac{2 A_{i}}{w}=1$. Figure $2 \mathrm{~b}$ (iv) shows that this process takes $<1 \mathrm{~s}$. Using an exponential fit, we find a time constant equal to $0.6 \pm 0.1 \mathrm{~s}$. A similar measurement, carried out with the turn-on of the heating at $t=8.9 \pm 0.1 \mathrm{~s}$, leads to a time constant of $0.25 \pm 0.15 \mathrm{~s}$ for the opening of the valve, a value substantially smaller than the closing time. One may speculate that these switching times can favorably be compared with the characteristic time $\frac{h^{2}}{D_{\text {gel }}}$ (Ref. 41), where $h$ is the channel height and $D_{\text {gel }} \sim 10^{-10} \mathrm{~m}^{2} / \mathrm{s}$, a plausible value for a diffusion constant in this context. From a practical prospective, the sub-second switching times we find are well adapted to biotechnological applications.

\section{Hydrogel-based caging}

Caging is a new functionality that is achieved by patterning hydrogel in the shape of square enclosures, as shown in Figure 3. This Figure shows the 3D topography of a $100 \times 100 \mu \mathrm{m}$ square cage, $3 \mu \mathrm{m}$-high in the dry state, confined in a microfluidic chamber, $10 \mu \mathrm{m}$-high, at different temperatures, above and below the LCST. At low temperature $\left(25^{\circ} \mathrm{C}\right)$, walls are raised, forming a cage that fills entirely the chamber height, while at higher temperature $\left(40^{\circ} \mathrm{C}\right)$, walls collapse down to $4 \mu \mathrm{m}$, opening the cage. The movie presented in Supplementary Information 5 shows the dynamics of opening and closing of such cages. Furthermore, the movie shown in the Supplementary Information 6 shows two cages separated by $300 \mu \mathrm{m}$ actuated independently.

Cages can be used to isolate entities, such as molecules, cells (see the following section), particles, and deliver them on demand them. For the sake of visualization, this functionality can be 


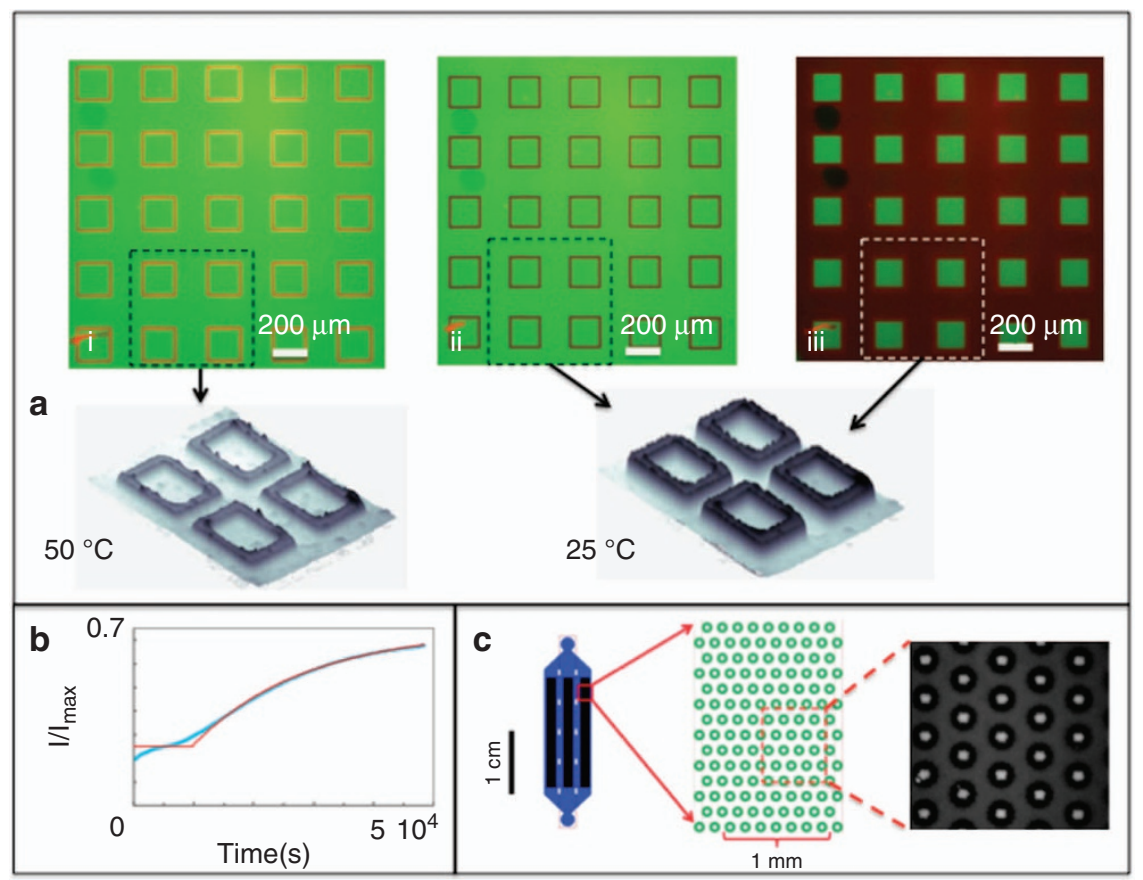

Figure 3 (a) Sequences of fluid injections and temperature changes for a system of 25 hydrogel cages, $200 \times 200 \mu \mathrm{m}$ in size with $10 \mu \mathrm{m}$ thick walls, in a microfluidic chamber with a dimension of $1 \mathrm{~cm} \times 2.5 \mathrm{~mm} \times 10 \mu \mathrm{m}$ along with the topographic images of a similar system including four cages. A fluorescein solution is driven through the system with walls in a low position (temperature held at $50{ }^{\circ} \mathrm{C}$ ) (i), with raised walls touching the upper wall of the chamber (ii). The system is washed by water while fluorescein is isolated in the closed chambers (iii). (b) Temporal increase of the fluorescence signal $I / I_{\max }$ ((blue line-see text) in a rectangular cage as fluorescein diffuses through the walls. The red line is the theoretical model using a diffusion constant $D=2.510^{-13} \mathrm{~m}^{2} \mathrm{~s}^{-1}$ through the gel (see Supplementary Information 7 for more details). (c) System similar to (a), Caging-functioning system with 3000 circular walls represented at various scales. The right figure shows fluorescein (white) trapped in the circular walls (black) in water (gray). The diameter of the cage is $100 \mu \mathrm{m}$, and the walls are $25 \mu \mathrm{m}$ wide. The density is 44 cages per $\mathrm{mm}^{2}$.

illustrated with fluorescein and rhodamine. In Figure 3a, twentyfive square cages $(10 \times 200 \times 200 \mu \mathrm{m})$ are integrated in a shallow NOA chamber through which different aqueous solutions are driven. First, the chamber is washed with a fluorescein solution and the temperature is increased to $50^{\circ} \mathrm{C}$. The cage walls (appearing as red in the figure due to rhodamine labelling) are in a low position. Then, the flow is maintained while the temperature is decreased: because the cages are thicker, they appear as dark zones, whereas the premises of photobleaching appear inside the cages as a result of the isolation and immobilization of the fluorescein (Figure 3a (ii)). Finally, Figure $3 a$ (iii) shows that, after flushing the system, the fluorescein is trapped in the cages. Isolation is efficient because, as shown in Figure $3 \mathrm{~b}$, there is no observable fluorescein exchange between the inside and the outside of the cages within the time scale we have considered (minutes). The fluorescein can later be released with further heating (shown in Supplementary Information 6).

The question of the permeability of the gel barrier is addressed in Figure $3 \mathrm{~b}$ and developed in Supplementary Information 7. We performed diffusion of fluorescein experiments through the walls of a single rectangular trap. In these experiments, fluorescein is initially outside the wall and we measure the kinetics of diffusion of this solute through the four walls of the cage by tracking the fluorescence intensity level of the fluorescein in the cage as a function of time, averaged over the cage area. The plot in Figure $3 \mathrm{~b}$ shows that the normalized intensity $\frac{1}{I_{\max }}$ (where $I_{\max }$ is the intensity outside the cage) increases with time but at a very slow rate- $10 \mathrm{~h}$ is required to reach a concentration comparable to that of outside the cage. By fitting the curve with a diffusion model (Supplementary Information 7), we find an effective diffusion constant of $2.5 \times 10^{-13} \mathrm{~m}^{2} / \mathrm{s}$, which is three orders of magnitude below the free diffusion constant of fluorescein. This slowing down of the diffusion process due to confinement (pore sizes are 1-2 nm, which is comparable to the fluorescein molecule), is consistent with models published in the literature ${ }^{42}$. This experiment shows that barriers can be used as membranes with controlled transport properties, a property potentially interesting for microbiology applications.

The concept of high density, that is, the capacity of the hydrogel technology to operate with a large number of cages, is illustrated in Figure 3c (see Supplementary Information 8 for details). Here, we produced a device with 3000 circular chambers and perform a series of experiments identical to those in Figure 3a. Figure $3 c$ shows the design of the device and an enlargement of the array containing 130 cages on a surface of $3 \mathrm{~mm}^{2}$, which is a density of 44 cages par $\mathrm{mm}^{2}$, that is, roughly one cage per $100 \mu^{2}$. This density is comparable to the state-of-the-art achieved with PDMS valves and droplet technology. The figure on the right shows the details of the system: the fluorescein is trapped in 23 cages similarly to Figure a (iii). This demonstrates that hydrogel caging technology enables the fabrication of functional high-density devices.

\section{Applications: single-cell trapping/release and DNA isothermal amplification}

In this section, we illustrate two applications of our hydrogel technology. The first, shown in Figure $4 a$, is the trapping and release of individual cells. Circulating B cells are injected in a chamber $10 \mu \mathrm{m}$ high, containing 8 square cages that are $300 \mu \mathrm{m}$ wide with $50 \mu \mathrm{m}$ thick hydrogel walls. In Figure $4 a$ (i), the cage is at an expanded state and several cells accumulate at the wall side exposed to the upstream current near the stagnation point. As the system heats up, the walls collapse and a flow above the walls is generated, freeing 


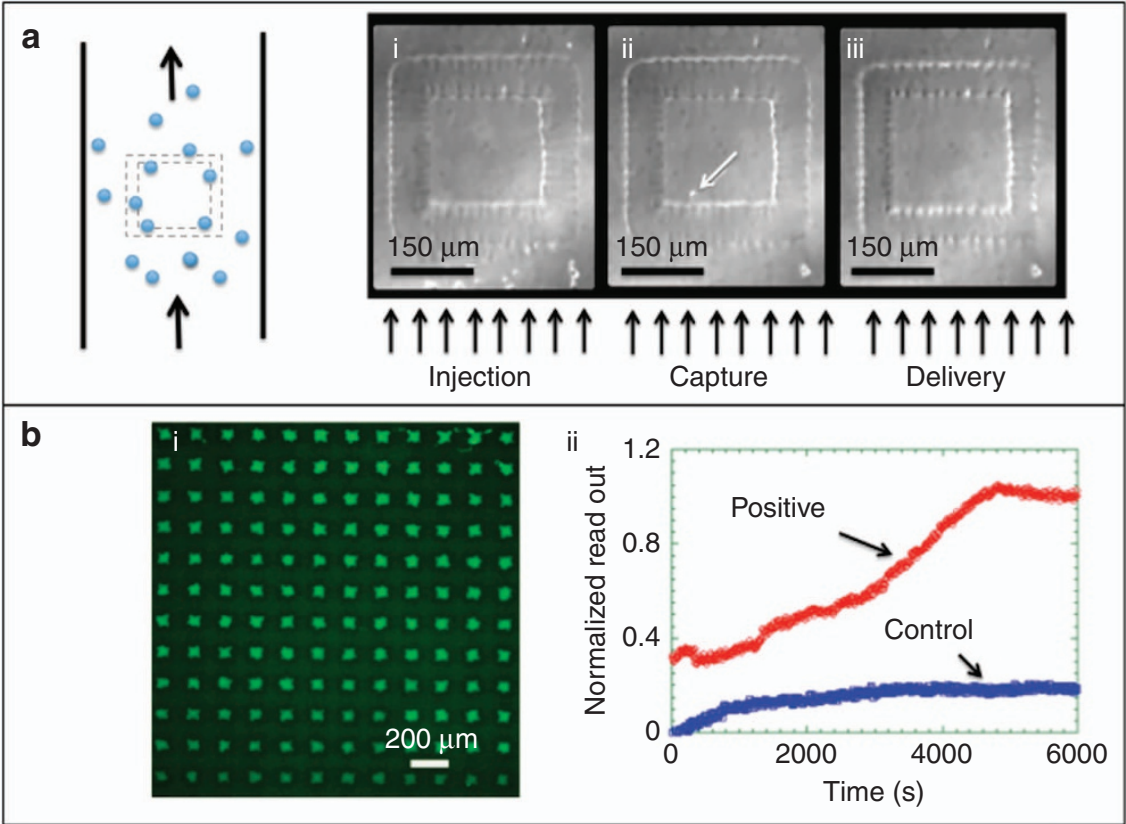

Figure 4 (a) Single cell capture by an individual cage ( $\mathrm{i}$ and ii) and release (iii). The chamber is $10 \mu \mathrm{m}$ high and $300 \mu \mathrm{m}$ wide with $50 \mu \mathrm{m}$ thick walls (dry thickness $3.5 \mu \mathrm{m}$ ). In (i), the cage is closed and a suspension of circulating B cells is introduced into the device. In (ii), the cage walls are lowered, then raised, to capture an individual cell (noted by the white arrow) In (iii), the cage is lowered again to release the cell. (b) (i) Image showing 144 cages in a contiguous system of 7800 cages three hours after filling the device with the sample (buffer containing the Human Synaptonjanin 1 gene and the RCA amplification mix). Each cage is $50 \mu \mathrm{m} \times 50 \mu \mathrm{m}$; the chamber height is $20 \mu \mathrm{m}$. (ii) The amplification curve extracted from intensity measurements in a device similar to (b (i)), but containing a negative control chamber. $t=0$ represents the time at which walls have been raised, $<5 \mathrm{~min}$ after the sample preparation. In practice, the fluorescence intensity is measured in two situations: cages filled with the mix and the DNA sample (positive), and cages filled with the mix and water instead of DNA (control), keeping the same mix concentration for the two experiments. The signal is normalized with the intensity measured at a time of $6000 \mathrm{~s}$. The signal is provided by a single cage with the intensity being integrated over the cage area. We checked that all the cages behave the same.

the accumulated cells. By lowering the temperature, a single cell can be trapped in the cage (Figure $4 a$ (ii)). This cell is released upon further heating (Figure 4a (iii)). Although the protocol is manual, it nonetheless illustrates the potential of the technology regarding capture and release of individual cells.

The application of our hydrogel technology to isothermal amplification and NAAT is illustrated in Figure 4b. Here, the system is filled with a biological sample composed of a buffer containing the Human Synaptojanin 1 gene at concentrations of $84 \mathrm{pg} \mathrm{\mu l}^{-1}$ and a mix designed for RCA amplification with primers targeting the gene. SYNJ1 mutations are suspected to be involved in several neurodegenerative diseases, such as Parkinson's disease (see Materials and Methods section). The goal of the experiment illustrated here is to detect its presence. Figure $4 b$ (i) shows 144 cages as part of a system of 7800 cages system after $3 \mathrm{hrs}$ of isothermal amplification. The latter is performed once the chip is filled with the sample and RCA mix at $40{ }^{\circ} \mathrm{C}$ and cooled down to $28^{\circ} \mathrm{C}$ to seal the cages. The image shows that the gene is amplified homogeneously in each cage. The presence of the hydrogel is not detrimental to RCA amplification. Figure $4 \mathrm{~b}$ (ii) shows a typical amplification curve along with a negative control containing no DNA. In the control experiment, a small fluorescence signal is produced by the probes. However, the signal over noise ratio remains important. From a NAAT prospective, one can conclude from the inspection of Figure $4 \mathrm{~b}$ that the gene is present in the sample. This system illustrates the diagnostic capability of our technology.

\section{DISCUSSION AND CONCLUSION}

In this paper, by using an ex situ hydrogel-based technology, we microfabricated devices with up to 7800 actuated micro-cages that sequester and release solutes, along with valves actuated individually with switching times of 250 and 600 ms for the opening and closing respectively. Two applications of this technology were illustrated in the domain of single cell handling and NAAT for the Human Synaptojanin 1 gene.

From a technological prospective, in the case of isothermal systems (all cages actuated at once), it is not difficult to achieve VLSI (Very large System Integration) by reducing the size of the hydrogel valves or cages to $5 \mu \mathrm{m}$. In this case, we can reach a density of 1 million valves or cages per $\mathrm{cm}^{2}$. With cages actuated independently, the situation is more challenging, but densities of thousands of cages per $\mathrm{cm}^{2}$ - still a high throughput - can certainly be envisioned.

Caging functionality allows the trapping of entities under high throughput conditions similar to droplet-based technology. However, since gels are permeable, hydrogel walls can also operate as membranes, allowing the exchange of small molecules between the sequestered entity (for example, cells) and the external medium.

From a general perspective, there is room to extend or diversify the range of functionalities of our hydrogel technology by varying the gel chemistry, using different stimuli (for example, light, $\mathrm{pH}$ ), developing different behaviors (LCST or UCST), or grafting molecules, exploiting specific affinities for analytical purposes.

The biotechnological applications of our responsive hydrogelbased technology are numerous. Digital amplification is an example. In our case, thousands of cages can be integrated on a single system so that high throughput screening or high sensitivity analyses of samples are feasible and with direct readout. Single-cell manipulation, stimulated by a growing recognition of the important role that play genomic heterogeneities in biological systems, provides another example of the applications of our technology. Microbiology is another field 
where thermo-sensitive hydrogels with well-controlled chemistry offer potential advantages. In this case, the permeability of the gel can be harnessed to deliver therapeutic molecules to cells trapped in cages to screen antibiotics. In addition, most importantly, these applications can be developed under interesting cost conditions because the materials are inexpensive and actuation requires low voltage sources or standard hot plates.

\section{ACKNOWLEDGEMENTS}

We gratefully thank the Institut Pierre-Gilles de Gennes (IPGG), the French National Research Agency (ANR), EQUIPEX and LABEX IPGG (Grant Nos. ANR-10-IDEX-0001-02, ANR-10-LABX-31,PSL*), the China Scholarship Council (CSC), ESPCI, CNRS, PSL, and the Ministry of Science and Technology of Thailand (MOST) for their financial support. We are grateful to Jacques Prost who initiated the collaboration. We are grateful for the help and discussions with Emilie Bayon, Ghimire Buwan, Luce Dauphinot, Olivier Mesdjian, Jacques Fattacioli, and Marie Caroline Jullien.

\section{COMPETING INTERESTS}

The authors declare no conflict of interest.

\section{REFERENCES}

1 Terry SC, Jerman JH, Angell JB. A gas chromatographic air analyzer fabricated on a silicon wafer. IEEE Transactions on Electron Devices 1979; 26: 1880-1886.

2 Oh KW, Ahn CH. A review of microvalves. Journal of Micromechanics and Microengineering 2006; 16: R13.

3 Unger MA, Chou H-P, Thorsen $\mathrm{T}$ et al. Monolithic microfabricated valves and pumps by multilayer soft lithography. Science 2000; 288: 113-116.

4 Thorsen T, Maerkl SJ, Quake SR. Microfluidic large-scale integration. Science 2002; 298: $580-584$.

5 Araci IE, Quake SR. Microfluidic very large scale integration (mVLSI) with integrated micromechanical valves. Lab on a Chip 2012; 12: 2803.

6 Cheow LF, Viswanathan R, Chin C-S et al. Multiplexed analysis of protein-ligand interactions by fluorescence anisotropy in a microfluidic platform. Analytical Chemistry 2014; 86: 9901-9908.

7 Wu AR, Kawahara TLA, Rapicavoli NA et al. High throughput automated chromatin immunoprecipitation as a platform for drug screening and antibody validation. Lab on a Chip 2012; 12: 2190.

8 Fordyce PM, Gerber D, Tran D et al. De novo identification and biophysical characterization of transcription-factor binding sites with microfluidic affinity analysis. Nature Biotechnology 2010; 28: 970-975.

9 Song $\mathrm{H}$, Chen DL, Ismagilov RF. Reactions in droplets in microfluidic channels. Angewandte Chemie International Edition 2006; 45: 7336-7356.

10 Guo MT, Rotem A, Heyman JA et al. Droplet microfluidics for high-throughput biological assays. Lab on a Chip 2012; 12: 2146.

11 Lagus TP, Edd JF. A review of the theory, methods and recent applications of high-throughput single-cell droplet microfluidics. Journal of Physics D: Applied Physics 2013; 46: 114005.

12 Sackmann EK, Fulton AL, Beebe DJ. The present and future role of microfluidics in biomedical research. Nature 2014; 507: 181-189.

13 Halperin A, Kröger $M$, Winnik FM. Poly( $N$-isopropylacrylamide) phase diagrams: fifty years of research. Angewandte Chemie International Edition 2015; 54: 15342-15367.

14 Döring A, Birbaum W, Kuckling D. Responsive hydrogels-structurally and dimensionally optimized smart frameworks for applications in catalysis, micro-system technology and material science. Chemical Society Reviews 2013; 42: 7391-7420.

15 Cohen Stuart MA, Huck WTS, Genzer J et al. Emerging applications of stimuliresponsive polymer materials. Nature Materials 2010; 9: 101-113.

16 Mendes PM. Stimuli-responsive surfaces for bio-applications. Chemical Society Reviews 2008; 37: 2512-2529.

17 Beebe DJ, Moore JS, Bauer JM et al. Functional hydrogel structures for autonomous flow control inside microfluidic channels. Nature 2000; 404: 588-590.

18 Richter A, Klatt S, Paschew $\mathrm{G}$ et al. Micropumps operated by swelling and shrinking of temperature-sensitive hydrogels. Lab on a Chip 2009; 9: 613-618.
19 Yu C, Mutlu S, Selvaganapathy P et al. Flow control valves for analytical microfluidic chips without mechanical parts based on thermally responsive monolithic polymers. Analytical Chemistry 2003; 75: 1958-1961.

20 Richter A, Kuckling D, Howitz S et al. Electronically controllable microvalves based on smart hydrogels: Magnitudes and potential applications. Journal of Microelectromechanical Systems 2003; 12: 748-753.

21 Wang J, Chen Z, Mauk M et al. Self-actuated, thermo-responsive hydrogel valves for lab on a chip. Biomedical Microdevices 7: 313-322.

22 Bäcker M, Raue M, Schusser S et al. Microfluidic chip with integrated microvalves based on temperature- and $\mathrm{pH}$-responsive hydrogel thin films. Physica Status Solidi A 2012; 209: 839-845.

23 Harmon ME, Tang M, Frank CW. A microfluidic actuator based on thermoresponsive hydrogels. Polymer 2003; 44: 4547-4556.

24 Son KJ, Shin D-S, Kwa T et al. A microsystem integrating photodegradable hydrogel microstructures and reconfigurable microfluidics for single cell retrieval. Lab on a Chip 2015; 15: 637-641.

25 Vosseler M, Clemenz M, Zengerle R. A flat and cost effective actuator based on superabsorbent polymer driving a skin attachable drug delivery system. Smart Materials and Structures 2012; 21: 105002.

26 Lin S, Wang W, Ju X-J et al. A simple strategy for in situ fabrication of a smart hydrogel microvalve within microchannels for thermostatic control. Lab on a Chipp 2014; 14: 2626-2634.

27 Kwon GH, Choi YY, Park JY et al. Electrically-driven hydrogel actuators in microfluidic channels: fabrication, characterization, and biological application. Lab on a Chip 2010; 10: 1604-1610.

28 Sugiura S, Sumaru K, Ohi K et al. Photoresponsive polymer gel microvalves controlled by local light irradiation. Sensors and Actuators A: Physical 2007; 140: 176-184.

29 Agarwal AK, Sridharamurthy SS, Beebe DJ et al. Programmable autonomous micromixers and micropumps. Journal of Microelectromechanical Systems 2005; 14: $1409-1421$.

30 Ionov L. Hydrogel-based actuators: Possibilities and limitations. Materials Today. 17:494-503.

31 Liu Z, Toh W, Ng TY. Advances in mechanics of soft materials: a review of large deformation behavior of hydrogels. International Journal of Applied Mechanics 2015; 07: 1530001.

32 Zhao X, Hong W, Suo Z. Inhomogeneous and anisotropic equilibrium state of a swollen hydrogel containing a hard core. Applied Physics Letters 2008; 92: 051904.

33 Marcombe R, Cai S, Hong $\mathrm{W}$ et al. A theory of constrained swelling of a pHsensitive hydrogel. Soft Matter 2010; 6: 784-793.

34 Hoffmann J, Plotner M, Kuckling $D$ et al. Photopatterning of thermally sensitive hydrogels microactuators. Sensors and Actuators 1999; 77: 139-144.

35 Chollet B, Li M, Martwong E et al. Multiscale surface-attached hydrogel thin films with tailored architecture. ACS Applied Materials and Interfaces 2016; 8: 11729-11738.

36 Duffy DC, McDonald JC, Schueller OJA et al. Rapid Prototyping of Microfluidic Systems in Poly(dimethylsiloxane). Analytical Chemistry 1998; 70: 4974-4984.

37 Bartolo D, Degré G, Nghe $\mathrm{P}$ et al. Microfluidic stickers. Lab on a Chip 2008; 8: 274-279.

38 Li M, Bresson B, Fretigny C et al. Submicrometric films of surface-attached polymer networks: temperature-responsive properties. Langmuir 2015; 31: 11516-11524.

39 Chollet B, Li M, Martwong E et al. Multiscale surface-attached hydrogel thin films with tailored architecture. ACS Applied Materials and Interfaces 2016; 8: 11729-11738.

40 Chollet B, D'Eramo L, Li M et al. Tailoring patterns of surface-attached multi-responsive polymer networks. ACS Applied Materials and Interfaces 2016; 8: 24870-24879.

41 Tanaka T, Fillmore DJ. Kinetics of swelling of gels. J Chem Phys 1979; 70: 1214.

42 Amsden B. Solute diffusion within hydrogels. Mechanisms and models. Macromolecules 1998; 31: 8382-8395.

This work is licensed under a Creative Commons Attribution 4.0 International License. The images or other third party material in this article are included in the article's Creative Commons license, unless indicated otherwise in the credit line; if the material is not included under the Creative Commons license, users will need to obtain permission from the license holder to reproduce the material. To view a copy of this license, visit http://creativecommons.org/licenses/ by/4.0/

(c) The Author(s) 2018

Supplementary Information for this article can be found on the Microsystems \& Nanoengineering website (http://www.nature.com/ micronano) 